The main problem for the solar collector is probably degradation of the collector surface by hot gas and dust ejected from the heated spot on the asteroid. This problem could be greatly ameliorated by the use of small, rugged secondary or tertiary mirrors near the asteroid, while the large primary collector could stand off some distance because of its long focal length (C. Steffens, personal communication).

\section{H. J. Melosh}

Lunar and Planetary Laboratory,

University of Arizona,

Tucson, Arizona 85721, USA

I. V. Nemchinov

Institute for Dynamics of Geospheres,

Russian Academy of Sciences,

Leninsky Prospekt 38, Bldg 6,

117334 Moscow, Russia

\section{Viral burden in AIDS}

SIR - The discussion ${ }^{1-3}$ about the interpretation of the reports of a large viral burden in the lymph nodes of asymptomatic people infected with HIV is an argument about details which in essence are not disputed by either side. Essentially, HIV is able to kill or delete CD4 function by several direct and indirect mechanisms. The diversity of this virus and its cytopathic prospectives in vitro suggest that disease should rapidly ensue following infection. The fact that it usually does not and that many longterm survivors, as well as chimpanzees, do not seem to be succumbing to the effects of HIV infection, strongly suggests that CD4 tropism is not sufficient.

Sheppard et al. ${ }^{1}$ are right in that immune activation is of paramount importance in determining disease outcome. In this regard, progression to AIDS has many features of disease of chronic activation, whether induced by viruses acting as superantigens (MMTV, MLV-Maids) or by chronic allogeneic stimuli ${ }^{4}$. One outcome of the generalized activation of the immune response is to increase the virus burden, therefore the mechanisms under debate are complementary. As several studies clearly show either a marked genetic predisposition or resistance to rapid HIV disease progression, and the virus load and pathogenicity can be separated in the SIV-infected macaque ${ }^{5}$ (a model for AIDS), the importance of the lymphoreticular viral burden cannot

1. Sheppard, H. W., Ascher, M. S. \& Krowka, J. F. Nature 364. 291 (1993).

2. McLean, A. \& Michie, C. Nature 365, 301 (1993)

3. Garry, R. F. \& Fermin, C. D. Nature 365, 302 (1993)

4. Dalgleish, A. G. in AIDS Research Reviews (eds Koff, W. C. Wong-Staal, F. \& Kennedy, R. C.) 73-93 (Dekker, New Wong-Staal,
York, 1993).

5. Dalgleish, A. G. Curr. Opin. Immun. 5(4) 608-614 (1993).

6. Cameron, P. U. etal. Clin. exp. Immun. 88, 226-236 (1992) merely be decided by simplistic mathematical models or by seeing how quickly CD 4 cells can be killed in the test tube. It is therefore important to identify the host factors for progression and to define exactly which cells are infected in the lymph nodes and whether important cells, such as the follicular dendritic cells, need to be infected in order to activate or infect large numbers of lymphocytes, as has been suggested by Cameron et al. ${ }^{6}$.

The question of whether activation drives disease progression or whether it is merely a result of virus replication is so important that it is imperative to conduct selective immunosuppressive experiments in SIV-infected macaques. Prevention of SIV-induced disease by presuppression of the macaques before a live SIV challenge would compel us to view the issues under discussion in a new light.

\section{A. G. Dalgleish}

\section{Department of Cellular and Molecular} Sciences,

St George's Hospital Medical School, CranmerTerrace,

London SW17 ORE, UK

SIR - It is becoming generally accepted that there is a larger viral load in HIVinfected individuals than hitherto thought. It is possible to calculate the total proviral load within an infected individual which, depending on the disease stage, is $>10^{6}-10^{11}$. But the proviral load provides a snapshot of events and gives little idea as to the dynamics of viral production and clearance. The viral load can be likened to a bucket standing under a tap: the limited size of the bucket is certainly important, but the consequences are ultimately determined by whether the tap was merely dripping or fully open. A wealth of data has described HIV infection in terms of chronic immune system activation: active polyclonal B-cell proliferation; intense HIV-specific cytotoxic T-lymphocyte effector frequencies; elevated peripheral CD 8 cell counts; elevated serum $\beta 2$ microglobulin; and urine neopterin, to cite just a few.

Variation of the nucleotide sequence of HIV or SIV may yield pertinent temporal information as it represents a cumulative indicator of past events. HIV nucleotide sequence diversity increases over time from, in most cases, a homogeneous origin to reach an intrapatient diversity of $>10 \%$. Longitudinal analyses of sequence diversity following experimental innoculation of macaques by SIV derived from molecularly cloned virus yielded nucleotide fixation rates of $\sim 1 \%$ per site per year ${ }^{1,2}$. Assuming a nucleotide substitution rate of $\sim 0.1-1$ per genome per cycle $^{3,4}$, approximately $500-5,000$ consecutive rounds of replication would be necessary to achieve the observed $10 \%$ genetic variation between any two proviruses sampled at the same time. If the viral burst size was merely two per cycle, a total of $2^{500}-2^{5,000}\left(10^{150}-10^{1,500}\right)$ would result, well in excess of the number of hydrogen atoms in the Universe! Only if the mean burst size was restricted a hundredfold or more $\left(1.004^{5,000} \sim 10^{9}, 1.04^{500}\right.$ $\sim 10^{8.5}$ ) could proviral loads approaching something like $10^{6}-10^{11}$ be achieved.

The most obvious explanation for the highly restricted viral burst would be an extraordinarily efficient clearance of virions and infected cells by the immune system. Most HIV-infected CD $4^{+} \mathrm{T}$ cells must therefore be cleared before virus can be produced, and loss of this cell type has always been the hallmark of HIV infection. The production of defective genomes could also limit amplification of HIV. But if the HIV mutation rate is $<1$ per genome per cycle, immune clearance is probably the dominant force in limiting the spread of HIV as opposed to the formation of defective viruses. Nonetheless, even though a defective provirus would be incapable of producing the full complement of viral proteins, as long as some were synthesized, the cell would be able to present HIV peptides in the context of MHC class I antigens, so attracting HIV-specific cytotoxic $\mathrm{T}$ cells - and $\mathrm{CD} 4^{+} \mathrm{T}$-cell destruction.

Very little is known about the dynamics of the human immune system. Certainly the lifespan of memory (CD45R0) and naive (CD45RA) $\mathrm{T}$ cells is different ${ }^{5}$. Germinal centres come and go depending on the presence of antigen. T-cell homeostasis would seem possible, but the mechanics and implications for HIV disease are hard to assess ${ }^{6}$. Of course more is known about the mouse, where $30-40 \%$ of peripheral immunocompetent $\mathrm{T}$ and $\mathrm{B}$ cells are renewed every 3 days $^{7}$. Much more information on the dynamics of the human immune system is needed.

Cumulative sequence variation reflects the repeated series of HIV/SIV $\mathrm{CD}^{+}$ $\mathrm{T}$-cell interactions and points to massive viral production and intense antiviral immune responses. Even though at any one time a minority of $\mathrm{CD}^{+} \mathrm{T}$ cells may be infected, given the numbers of replication cycles involved, and of course the time to accomplish these cycles, perhaps it is not too surprising that the immune system ultimately loses.

\section{Simon Wain-Hobson}

Unité de Rétrovirologie Moléculaire, Institut Pasteur,

28Rue du DrRoux,

75724 Paris cedex 15, France

1. Burns, D. P. W. \& Desrosiers, R. C. J.Virol. 65,1843 1854 (1991)

2. Johnson, P. R. et al. Virology 185, 217-228(1991)

3. Pathak, V. K. \& Temin, H. M. Proc natn. Acad. Sci. U.S.A 87, 6019-6023 (1990)

4. Leider, J. M., Paiese, P. \& Smith, F. I. J. Virol. 62 3084-3091 (1988)

5. Michie, C. et al. Nature 360, 264-265 (1992)

6. Margolick, J. B. et al. J. AIDS6, 153-161 (1993)

7. Freitas, A. A. \& Rocha, B. B. Immun. Today 14, 25-29 (1993).

NATURE · VOL 366 • 4 NOVEMBER 1993 